\title{
Reduced Spatial Resolution MRI Suffices to Image and Quantify Drought Induced Embolism Formation in Trees
}

\section{Marco Meixner}

Technical University Munich: Technische Universitat Munchen

\section{Petra Foerst}

Technical University Munich: Technische Universitat Munchen

Carel Windt ( $\nabla$ c.windt@fz-juelich.de)

Forschungszentrum Jülich https://orcid.org/0000-0003-2400-5407

\section{Research}

Keywords: Magnetic resonance imaging, MRI, NMR, Spatial resolution, Xylem, Embolism, Vulnerability curve, Small-scale, Low field

Posted Date: December 1st, 2020

DOl: https://doi.org/10.21203/rs.3.rs-112909/v1

License: (c) (i) This work is licensed under a Creative Commons Attribution 4.0 International License. Read Full License

Version of Record: A version of this preprint was published at Plant Methods on April 6th, 2021. See the published version at https://doi.org/10.1186/s13007-021-00732-7. 


\section{Reduced spatial resolution MRI suffices to image and quantify drought induced embolism formation in trees}

2 Marco Meixner ${ }^{1,2}$, Petra Foerst ${ }^{1}$, Carel W. Windt ${ }^{2 *}$

$3{ }^{1}$ Chair of process systems engineering, Technical University Munich, Munich, Germany

$4 \quad 2$ IBG-2: Plant Sciences, Forschungszentrum Jülich, Jülich, Germany

5 * Correspondence:

6 Carel Windt

7 c.windt@fz-juelich.de

8

9

10

11

12

13

14

15

16 
17

Background: Magnetic resonance imaging (MRI) is uniquely suited to non-invasively and continuously monitor embolism formation in trees. Depending on the MRI method used, quantitative parameter maps of water content and MRI signal relaxation behavior can be generated. The ability to measure dynamic differences in water content and relaxation behavior can be used to detect xylem embolism formation, even if xylem conduits are too small to be spatially resolved. This is especially advantageous when using affordable small-scale low-field MRI scanners. The amount of signal that can be obtained from an object strongly depends on the strength of the magnetic field of the imager's magnet. Imaging at lower resolutions thus would allow to reduce the cost, size and weight of the MRI scanner and to shorten image acquisition times.

Results: We investigated how much spatial resolution can be sacrificed without losing the ability to monitor embolism formation in coniferous softwood (spruce, Picea abies) and diffuse porous beech (Fagus sylvatica). Saplings of both species were bench dehydrated, while they were continuously imaged at stepwise decreasing spatial resolutions. Imaging was done by means of a small-scale MRI device, utilizing image matrix sizes of $128 \times 128,64 \times 64$ and $32 \times 32$ pixels at a constant FOV of 19 and $23 \mathrm{~mm}$, respectively. While images at the lowest resolutions (pixel sizes $0.59 \times 0.59 \mathrm{~mm}$ and $0.72 \times 0.72 \mathrm{~mm}$ ) were no longer sufficient to resolve finer details of the stem anatomy, they did permit an approximate localization of embolism formation and the generation of accurate vulnerability curves.

Conclusions: When using MRI, spatial resolution can be sacrificed without losing the ability to visualize and quantify embolism formation. Imaging at lower spatial resolution to monitor embolism formation has two advantages. Firstly, the acquisition time per image can be reduced dramatically. This enables continuous imaging at high time resolution, which may be 
beneficial to monitor rapid dynamics of embolism formation. Secondly, if the requirements for spatial resolution are relaxed, much simpler MRI devices can be used. This has the potential to make non-invasive MR imaging of embolism formation much more affordable and more widely available.

Keywords: Magnetic resonance imaging, MRI, NMR, Spatial resolution, Xylem, Embolism, Vulnerability curve, Small-scale, Low field

\section{Background}

During the last couple of decades two non-invasive imaging methods emerged that allow the visualization and quantification of embolism formation and spread in plant xylem: Highresolution computed tomography (HRCT, also referred to as micro CT) and MRI. For both imaging modalities methods have been developed to determine the percentage loss of xylem conductivity (PLC) due to the cavitation of xylem conduits. This is done by measuring, directly or indirectly, the amount of conducting xylem area that is lost.

HRCT currently is the most widely used method, known for the excellent spatial resolution it affords. It is capable of resolving individual xylem conduits, even in tree species with small conduit diameters. This allows to determine what conduits were filled before dry-down, which conduits cavitate during dry-down, and to estimate their conductivity (and loss thereof) on the basis of their diameters, in combination with the Hagen-Poiseuille law. This has been done for Eucalyptus camaldulensis [1], laurel [2], walnut [3], as well as P. tremula x P. alba P. pinaster and Q. robur [4]. An important drawback of HRCT, however, is the use of ionizing radiation. Depending on the dosage, it may damage living tissue [5], disrupt cellular function [6] and cause growth inhibition [7]. Therefore, while it is an excellent tool to visualize embolized conduits in high spatial detail, it is not an ideal choice to monitor dynamic processes that require repeated or long-term imaging with large numbers of exposures. 
For the latter purpose MRI, on the other hand, is especially well suited. It does not rely on the absorption of ionizing radiation by the sample, but is based on the principle of nuclear magnetic resonance (NMR). The energy deposition that is associated with MRI of small samples such as plant stems is negligible. Therefore, samples can be imaged non-invasively, for periods of months and with a virtually unlimited number of image acquisitions. Examples of such-long term experiments are imaging of flow and water distribution in a tree stem over a period of multiple seasons [8] or imaging of sap flow towards a developing tomato truss [9]. Using the appropriate hardware and imaging pulse sequences, parameter maps can be obtained that quantitatively represent NMR parameters such as amplitude (A) and $T_{2}$ [10]. The amplitude maps acquired in the current study thus linearly and quantitatively reflect water content, and are not merely weighted by it (for further details see $[10,11]$ ).

MRI usually cannot match the resolution offered by HRCT. The resolution that can be achieved by means of MRI is limited by the size of the sample, which usually dictates the smallest field of view (FOV) that can be used, and the number of pixels that can be acquired in that FOV. Reducing the FOV to zoom in on parts of an object in most cases is not possible, as signal from parts of the object that are outside of the FOV would fold back in, corrupting the image. The number of pixels (voxels) that can be encoded in a FOV is then defined by the strength and homogeneity of the magnetic field, as well as the strength of the imaging gradients, but almost never is chosen higher than $512 \times 512$. For plants matrix size typically is $256 \times 256$ or lower (for a more detailed explanation of the tradeoffs involved, please see the theory section). This means that in practice, even for samples measuring no more than $10 \mathrm{~mm}$ in diameter, it will be hard to exceed an in-plane resolution of $40 \times 40 \mu \mathrm{m}$. MRI can thus only resolve xylem conduits in species or organs with wide vessels, as has been demonstrated for woody lianas [12], grapevine [2, 13-15], cucumber [16] and roots of maize [17]. 
To circumvent the problems imposed by this resolution limit, various techniques were developed to detect xylem embolism formation indirectly. In plants, the MR signal almost exclusively originates from protons of water molecules. This fact can be used to generate parameter maps that quantitatively reflect water content, relaxation behavior, diffusion or flow, or parameter maps that are merely weighted by one or more of these parameters. PLC has been calculated or estimated on the basis of parameter maps in a number of ways. One approach is to base PLC on the change in pixel brightness of water content weighted MR amplitude images [18]. PLC values have also been derived from binarizing various types of MR parameter maps. In the binarization approach the loss of xylem conductivity is estimated from the decline in the number of pixels above a chosen threshold, plotted in dependence of the xylem water potential. Binarization has been applied to different types of MR parameter maps, such as signal intensity maps [18-20] or quantitative water content maps [14]. In Meixner et al. [11] we obtained PLC values of beech saplings on the basis of binarizing $A * T_{2}$ product images, obtained by pixelwise multiplication of water content images $(A)$ and signal relaxation time images $\left(T_{2}\right)$. In $A * T_{2}$ product images even low numbers of filled xylem vessels per pixel easily became detectable, as well as their disappearance due to cavitation; and the binarization of $\mathrm{A}^{*} \mathrm{~T}_{2}$ product images resulted in accurate xylem vulnerability curves. Using this approach, detection of embolism formation and the quantification of xylem vulnerability curves could be done, despite the fact that pixels were five times larger than the average conduit diameter in beech.

There are two important benefits that may be had by imaging xylem dry-down at a lower than usual spatial resolution: MRI hardware can be scaled down in size and weight, and images can be acquired more rapidly. We recently demonstrated that imaging embolism formation is possible, even when using a mobile small-scale, low-field MRI scanner [11, 21]. The imagers used in these cases were based on a $16 \mathrm{~kg}$ permanent magnet. Integrated onto a trolley, the whole system weighed $45 \mathrm{~kg}$ and could easily be handled by a single person, even in the field. 
While this system can already be considered mobile, reducing spatial resolution would enhance mobility even further. If resolution is reduced, a lower magnetic field strength suffices to achieve the same signal to noise ratio (SNR), allowing lighter and cheaper permanent magnets to be used. A reduction in resolution would also allow the use of lighter and less powerful gradient amplifiers and therefore permit the development of low-cost imaging equipment that is even more lightweight and mobile. Another advantage when reducing spatial resolution is the ability to image more rapidly, which allows the acquisition of more PLC values per unit time, permitting higher accuracy when fitting vulnerability curves [22]. In addition, high temporal resolution dry-down movies can be generated which allow the investigation of embolism spread within and between annual rings [14, 19], even when this spread proceeds rapidly [18].

In this study we investigate if MRI spatial resolution can be reduced significantly, without losing the ability to detect and quantify embolism formation. To this end, we bench dehydrated spruce (Picea abies) and beech (Fagus sylvatica) saplings in a small-scale MRI system. During dry-down the plants' main stems were imaged continuously at three stepwise decreasing spatial resolutions. We tested whether timing and location of embolism formation during dry-down can be monitored and vulnerability curves can be obtained, even if pixel sizes are much (up to thirty times) larger than the average xylem conduit diameter.

\section{Theory: Benefits of imaging at lower spatial resolutions}

MR imaging experiments always involve a trade-off between spatial resolution, signal-to-noise ratio (SNR) and imaging time. The benefits of using a lower spatial resolution are illustrated by the following equation [23]: 


$$
\mathrm{SNR} / \text { pixel } \propto \mathrm{A}_{\mathrm{pix}} \mathrm{B}_{0}\left(\frac{\mathrm{N}_{\mathrm{avg}}}{\mathrm{SW} /\left(\mathrm{N}_{\mathrm{fq}} * \mathrm{~N}_{\mathrm{ph}}\right)}\right)^{1 / 2}
$$

Neglecting signal decay over time due to spin-spin or spin-lattice interactions, Eq. 1 illustrates how increasing the pixel area $\left(A_{p i x}\right.$, i.e. decrease matrix size $N_{f q} \times N_{p h}$ at constant FOV) can compensate for: (i) the use of magnets of a lower magnetic field strength $\left(\mathrm{B}_{0}\right)$; (ii) imaging with a lower number of averages $\left(\mathrm{N}_{\mathrm{avg}}\right)$; or (iii) imaging at higher spectral width (SW). If lower magnetic fields are permitted, the magnets that provide the main magnetic field can become smaller and lighter.

Alternatively, imaging at lower spatial resolutions would also allow measurements with higher temporal resolution. On the one hand because at lower resolution the number of phase encoding steps $\left(\mathrm{N}_{\mathrm{ph}}\right)$ is lower, but also because the number of averages $\left(\mathrm{N}_{\mathrm{avg}}\right)$ can be lowered, decreasing image acquisition time. Imaging at higher spectral width (SW) also facilitates mobile imaging in the field. It allows the use of magnets of lower homogeneity and ensures tolerance against the effects of temperature changes and magnet handling on the magnet's homogeneity during transport and field use $[11,24]$.

Apart from the static and homogeneous magnetic field $\left(\mathrm{B}_{0}\right)$ magnetizing the sample, MRI also requires switched magnetic field gradients to spatially encode the MR signal. The frequency width of a pixel $(\Delta v)$ is determined by the gradient strength $(\mathrm{G})$, the pixel width in real space $(\Delta r)$ in the encoding direction and the gyromagnetic constant for hydrogen $(\gamma)$

$$
\Delta v=\gamma \mathrm{G} \Delta \mathrm{r}
$$

The minimum value that is acceptable for $\Delta v$ is, amongst other factors, determined by the homogeneity of the magnet. The more homogeneous the magnet, the lower $\Delta v$ is allowed to be. Small-scale magnets that are exposed to temperature changes and handling, however, require 
higher $\Delta v$ values and therefore more powerful imaging gradients (G). The need for strong and fast gradient pulses in turn limits the mobility of the MR imager. Strong and fast gradient pulses require large and heavy gradient amplifiers. For mobile systems this is particularly relevant, since the gradient amplifiers constitute the largest and heaviest hardware components of an imager, besides the magnet. Imaging at lower spatial resolutions (higher values of $\Delta r$ ) hence allows for the use smaller, lighter, cheaper and more mobile gradient amplifiers.

\section{Materials and Methods}

\section{Plant materials and treatment}

A potted 3 year old spruce sapling (Picea abies, $82 \mathrm{~cm}$ high, $16 \mathrm{~mm}$ stem diameter at measurement position) was purchased from a local nursery (Pflanzenwelt Biermann, Trangstedt, Germany), as was a 3 year old beech sapling (Fagus sylvatica, $161 \mathrm{~cm}$ high, $16 \mathrm{~mm}$ stem diameter at measurement position, Baumschule Leonhard Veith, Merzenich-Golzheim, Germany). Both trees were subsequently grown in a greenhouse at $20^{\circ} \mathrm{C}$ day $(16 \mathrm{~h})$ and $16^{\circ} \mathrm{C}$ night $(8 \mathrm{~h})$ temperature, provided with supplemental light $(250 \mathrm{~W}$, Philips IP65 metal halogen vapor lamps) and kept well-watered by means of drip irrigation.

For the dry-down treatment the trees were moved to the lab. MRI scanning was started when the tree was still intact, for the spruce tree 11 hours and for the beech tree 9 hours prior to the initiation of dry-down, which was done by cutting the stems $5 \mathrm{~cm}$ above the soil. During the entire experiment the trees were illuminated by a sodium vapor lamp (SON-T Agro 400W, Philips) and kept at a temperature of $22^{\circ} \mathrm{C}$ during the day $(12 \mathrm{~h})$ and $18^{\circ} \mathrm{C}$ at night $(12 \mathrm{~h})$. The dry-down experiment was continued until the xylem was completely embolized, which took 47 $\mathrm{h}$ in case of spruce and $18 \mathrm{~h}$ for beech. The spruce dry-down experiment was performed in the first half of February 2019, the beech dry-down experiment in the middle of June 2019. 
The MRI scanner comprised a custom, small-scale C-shaped permanent magnet $(0.25 \mathrm{~T}, 16$ $\mathrm{kg}$ ), a custom plane parallel gradient set with a maximum gradient strength of $0.3 \mathrm{~T} / \mathrm{m}$ and a Kea II spectrometer (Magritek, Wellington, New Zealand), equipped with a built-in $100 \mathrm{~W}$ RF amplifier. The gradient set was driven by three BAFPA40 gradient amplifiers (BRUKER, Rheinstetten, Germany). Inside the gradient system a $20 \mathrm{~mm}$ solenoidal RF coil with tuning mechanism was mounted. For full details on the system see Meixner et al. [11].

After winding the radio frequency (RF) coil around a Teflon former, placed on the stem (at heights of 37 and $78 \mathrm{~cm}$ for spruce and beech respectively), the plants were slid into the probe head of the MRI scanner. Both trees were fixed relative to the imager by means of three metal clamps, positioned below and above the magnet, and near the position where the tree was cut. For both measurements, a glass reference tube with an inner diameter of $1.7 \mathrm{~mm}$, filled with nickel nitrate doped water and a $\mathrm{T}_{2}$ value of $112.4 \mathrm{~ms}$, was attached to the stem inside of the RF coil. To minimize external RF noise the magnet was covered with an earthed silver-plated copper mesh.

A multi-spin echo (MSE) pulse sequence was employed, acquiring 64 echoes with an echo time of $4 \mathrm{~ms}$ for the first echo and $2.1 \mathrm{~ms}$ for all subsequent ones. For all measurements, the spectral width was $100 \mathrm{kHz}$, the slice thickness $5 \mathrm{~mm}$, the dwell time $10 \mu \mathrm{s}$, the number of averages 4 and the repetition time $1.5 \mathrm{~s}$. The field of view (FOV) was $19 \times 19 \mathrm{~mm}$ for spruce and $23 \times 23$ $\mathrm{mm}$ for beech. Both plants were imaged continuously, acquiring sets of three images of increasing matrix size $(32 \times 32,64 \times 64$ and $128 \times 128$ pixels $)$ and measurement time $(3.5,7.2$ and 14.6 minutes, see Fig. 1). The resulting in-plane resolutions in $\mathrm{mm}$ were $0.59 \times 0.59,0.30 \times 0.30$ and $0.15 \times 0.15$ for spruce, and $0.72 \times 0.72,0.36 \times 0.36,0.18 \times 0.18$ for beech, respectively. 
with different spatial resolutions, each with the same temporal resolution of 25.3 minutes (Fig. 1).

\section{Data processing}

Especially the higher resolution images (matrix sizes $64 \times 64$ and $128 \times 128$ ) that were acquired as described above, due to the low field strength of the imager, were of lower SNR than desired for our purposes (Additional file 1). In order to raise SNR, a sliding window averaging approach was applied, averaging up to eight consecutive measurements of the same spatial resolution. Prior to sliding window averaging all images were corrected for small position changes of the tree during the dry-down. This was done by pixel-wise shifting each image, minimizing the difference between the shifted image and the first image of each time series. After sliding window averaging all resulting images were phase corrected according to Ma et al. [25]. Subsequently, quantitative water content $(A)$ and signal relaxation $\left(T_{2}\right)$ maps were obtained by mono-exponentially fitting the echo train of each image of the measurement time series in a pixel-by-pixel manner [26]. Prior to fitting, handmade masks were applied which only included the tree stem. $A^{*} T_{2}$ parameter maps were calculated by multiplying amplitude $(A)$ and $T_{2}$ images, again pixel-by-pixel (Fig. 2).

When generating dry-down movies via sliding window averaging of one, two, four or eight images, fitting errors in the form of erroneous, bright pixels were filtered out. This was done by setting pixels to zero that have double or more, or half or less the value of all four nearest

222 neighboring pixels.

\section{Calculation of vulnerability curves}

To generate vulnerability curves (VCs), the decline in xylem functionality was estimated from $\mathrm{A} * \mathrm{~T}_{2}$ product images and plotted as a function of stem water potential $\left(\Psi_{\text {stem }}\right)$. The latter was measured with a Scholander Chamber (Model 1000, PMS Instrument Company, Albany, USA). 
Throughout the dry-down, randomly chosen small twigs were carefully bagged with cling foil and aluminum foil (the latter to put the leaves in complete darkness) and left to equilibrate for 15 minutes. The twigs were then excised, quickly transferred to the instrument and measured. For spruce, 61 water potential values were measured over the course of four days, whereas during the more rapid dry-down of beech 26 water potential values were taken over a period of 26 hours.

During the dry-down experiments, the percentage of embolized xylem conducting area was obtained by binarizing $\mathrm{A}^{*} \mathrm{~T}_{2}$ product images. This was done by matching the center of the sliding window temporally to the water potential measurements and counting the number of pixels above the threshold $\mathrm{P}_{\text {abv. }}$ The binarization threshold was chosen via optimizing for a maximum $r^{2}$ value of the sigmodial fit (see below). For the different imaging matrices $(32 \times 32$, $64 \times 64,128 \times 128)$ the respective threshold values for spruce were $0.2,0.1,0.1$, and for beech 1.4, 1.3, 1.3. After binarization, the $\mathrm{P}_{\mathrm{abv}}$ values were normalized from 0 to $100 \%$ according to $\%_{\mathrm{emb}}=100 * \frac{\mathrm{P}_{\text {initial }}-\mathrm{P}_{\mathrm{abv}}}{\mathrm{P}_{\text {initial }}-\mathrm{P}_{\min }}$, with $\mathrm{P}_{\mathrm{abv}}$ referring to the initial and $\mathrm{P}_{\min }$ to the minimum number of pixels above the binarization threshold of a time series of images. The $\mathrm{VC}$ was obtained by fitting a sigmodial function in dependence of the measured xylem water potential $\left(\Psi_{\text {xylem }}\right)$ : $\%_{\mathrm{emb}}\left(\Psi_{\mathrm{xylem}}\right)=100 /\left(1+\exp \left(\frac{\mathrm{s}}{25}\left(\Psi_{\mathrm{xylem}}-\Psi_{50}\right)\right)\right) \quad[22]$, with the fit parameters indicating how quickly the dry down progressed through the xylem (slope: S) and the water potential at which $50 \%$ of the initially filled xylem were embolized $\left(\Psi_{50}\right)$. Normalization and the sigmodial fitting were performed with Matlab (Mathworks, Natick, USA). Fitting errors were calculated as described by Press [27].

\section{Microscopy}

After the dry-down experiment, the imaged stem pieces were marked, excised and stored in $75 \%$ ethanol (after previously equilibrating them in $25 \%$ and $50 \%$ ethanol for 24 hours each). 
Transverse sections of $20 \mu \mathrm{m}$ were prepared with a sliding microtome (GSL1, Schenkung Dapples, Zürich, Switzerland) and stained with a 1:1 (v/v) mixture of safranin and astrablue, following Gärtner and Schweingruber [28]. Images of the cross section were obtained with a digital camera (DFC 320, Leica, Cambridge, UK), connected to a light microscope (DM2500, Leica Microsystems GmbH, Wetzlar, Germany).

\section{Results and Discussion}

\section{What xylem anatomical detail can be resolved at decreasing spatial resolution?}

In spruce, water filled xylem could easily be distinguished from cambium and bark, even at a matrix size of 32×32 (Fig. 2a). Individual year rings, however, were only resolved at matrix sizes of $64 \times 64$ and above. The cambium is the brightest tissue in the $T_{2}$ images, where it showed maximum contrast against the surrounding tissue. The border between cambium and xylem, however, appearing as a black ring both in water content (amplitude, $\mathrm{A}$ ) and $\mathrm{T}_{2}$, was only resolved at matrix sizes of $64 \times 64$ and $128 \times 128$. The homogeneous distribution of water inside the filled xylem was similar to that previously imaged in pine stems [20, 29].

In beech, at all matrix sizes the filled xylem could be distinguished from the cambium due to a dark region between cambium and filled xylem (Fig. 2b). As in spruce, individual year rings could only be recognized at matrix sizes of $64 \times 64$ and above. In the microscopic images of beech (Fig. 4b), the sapling showed two complete growth rings, and a narrow region of early wood of a third growth ring inside of the cambium, which had formed during the current year. At all matrix sizes xylem containing filled vessels could be distinguished from non-filled xylem on the basis of higher water contents (40 to $60 \%$ ), but could be identified even more clearly by long $\mathrm{T}_{2}(60$ to $80 \mathrm{~ms})$ and $\mathrm{A}^{*} \mathrm{~T}_{2}$ values. Filled xylem was not found in all year rings, but only became visible in the middle of the second growth ring. Comparable water content and $\mathrm{T}_{2}$ values for filled and embolized xylem in beech were found in Meixner et al. [11]. Merela et al. 
[30] observed similar water content distribution patterns in the stem of a beech sapling (please note that in this example the amplitude images were water content weighted, but did not reflect water content quantitatively). The absence of filled vessels in the newly formed xylem may indicate that the plant experienced drought stress [31], or had been repotted in spring 2019.

Most of the finer details of the xylem anatomy of both beech and spruce thus were already resolved at a matrix size of $64 \times 64$ (pixel size of $0.30 \mathrm{~mm}$ in spruce and $0.36 \mathrm{~mm}$ in beech) and matrix sizes of $32 \times 32$ were sufficient to identify filled xylem in spruce and beech.

\section{Spatio-temporal characterization of the xylem dry-down at decreasing spatial resolution}

The $\mathrm{A} * \mathrm{~T}_{2}$ product images provided an effective means to monitor dry-down for all three matrix sizes in both xylem anatomies (Fig. 3a,b). In Meixner et al. [11] it was shown that both water content and $T_{2}$ images can be used to detect xylem cavitation in beech, but that $T_{2}$ maps are especially sensitive to it. By multiplying the parameter maps of water content (A) and $T_{2}, A * T_{2}$ product maps were obtained, in which regions with filled vessels in beech could be identified even more clearly. In the current study we show that in coniferous wood the same approach is effective as well. The presence of filled xylem conduits gave rise to bright $A * T_{2}$ regions, just like in beech, while dry-down manifested itself as a strong decrease in $A^{*} T_{2}$ values (Fig. 3a). In the following the dry-down of both species was therefore monitored on the basis of $\mathrm{A}^{*} \mathrm{~T}_{2}$ maps.

On the basis of $\mathrm{A}^{*} \mathrm{~T}_{2}$ product maps in spruce it was found that, independent of matrix size, the first emboli could be distinguished at a water potential of $-3.15 \mathrm{MPa}, 11.4 \mathrm{~h}$ after dry-down initiation (Fig. 3a). Embolism formation started in the current annual ring and at the border of the neighboring second ring (Fig. 3a, arrow SP1). From this region emboli spread, first radially and then tangentially within the outer annual ring (arrow SP2), and after $16.5 \mathrm{~h}$ emboli also started forming in the neighboring ring. Another detail that was visible at all three spatial 
resolutions was a narrow strip of xylem that remained hydrated in the outer annual ring, while the xylem tissue surrounding it appeared empty (arrow SP3). After $46.9 \mathrm{~h}$ (-3.8 MPa) nearly all xylem was embolized (Fig. 3a).

In beech, emboli started forming only a few hours after cutting the plant at its base (Fig. 3b). Embolism formation manifested itself in the form of a gradual decline in $\mathrm{A} * \mathrm{~T}_{2}$ pixel intensities. Filled xylem, which was found only in the middle of the second growth ring, cavitated within a short time window, between hour 7.5 and 12.0. Dry-down appeared gradual and spread uniformly over the entire filled xylem, indicating that vessels cavitated in an independent, rather than in a clustered manner. Embolism spread in a tangential and radial pattern, as observed by Meixner et al. [11] within beech, was not detected.

On the basis of difference images, even at the lowest spatial resolution, it was possible to detect embolism events that play out in a time window of only a few hours (see $\Delta_{-}$-maps in Fig. 3c,d). In spruce it was shown that the onset of embolism formation, which took place in the early wood of the outer annual ring, happened between hour 5 and 8 after initiation of the dry-down (Fig. 3c). In beech at the lowest spatial resolution it could be shown that the final phase of the xylem dry-down happened between hour 9 and 12 (Fig. 3d). A striking difference between the sequences of $\mathrm{A}^{*} \mathrm{~T}_{2}$ product images of both species was that in the xylem of spruce almost no intensity was left in the images after complete dry-down, whereas in beech always a significant amount of background signal was left visible. This reflects the differences in xylem anatomy of spruce and beech. In spruce, the xylem for the largest part consists of tracheids, with very little in between [32] (Fig. 4). The amount of remaining water in the tracheid walls was either too little to be recognized, or its $\mathrm{T}_{2}$ value was too short to be detected. In beech, to the contrary, the bulk of the xylem consists of narrow fibers with thick walls, interspersed between the vessels (Fig. 4). This pool of water remained visible and, with an average $\mathrm{T}_{2}$ value of $\sim 25 \mathrm{~ms}$, was detected in the stem, even when surrounding vessels cavitated. 
For an animated illustration of the observations mentioned in this section a tableau of nine videos are provided for both plants as supplementary material (Additional files $3 a$ and $b$ ).

\section{Are low resolution images sufficient to obtain xylem vulnerability curves?}

For spruce and beech, xylem vulnerability curves (VCs) were successfully obtained via binarizing $\mathrm{A}^{*} \mathrm{~T}_{2}$ product images of all three spatial resolutions. For both species, the binarization masks at different matrix sizes were consistent in the location and timing where xylem desiccation was observed (Fig. 5a,b). VCs based on those binarization masks showed $\mathrm{r}^{2}$ values $>0.90$ and yielded comparable $\Psi_{50}$ values. At matrix sizes of $32 \times 32,64 \times 64,128 \times 128$ for spruce values of $-3.8,-4.0$ and $-4.0 \mathrm{MPa}$ were found, for beech $-3.4,-3.5$ and $-3.5 \mathrm{MPa}$, respectively (Fig. 5c-h).

The $\Psi_{50}$ values found for beech at the different matrix sizes were in close agreement with one another, as well as with values obtained in other studies on beech saplings (Caquet et al. [33]: -3.0 to $-4.0 \mathrm{MPa}$; Aranda et al. [34]: -3.0 to $-3.7 \mathrm{MPa}$; Meixner et al. [11]: -3.2 to $-3.8 \mathrm{MPa}$ ) and mature beech trees (Lemoine et al. [35]: -2.2 to $-3.1 \mathrm{MPa}$; Herbette et al. [36]: -3.0 MPa; Schuldt et al. [37]: -3.3 to $-3.7 \mathrm{MPa}$; Stojnic et al. [38]: -2.9 to 3.5 MPa; Tomasella et al. [39]: -2.5 MPa). For spruce, the $\Psi_{50}$ values obtained in the current study were slightly higher than those obtained in other studies on spruce saplings (Mayr et al. [40]: -4.38 MPa; Chmura et al. [41]: -4.27 MPa), but lower or in agreement with values found for mature trees (Cochard [42]: -3.5 MPa; Tomasella et al. [39]: -4.01 MPa). Hence, we conclude that xylem dry-down can be quantified accurately on the basis of MR images, even if their spatial resolution is low and pixels are much larger than xylem conduit diameters.

\section{Conclusions}

In this study we demonstrated that MR imaging at low spatial resolution (pixel sizes $>0.5 \times 0.5$ $\mathrm{mm}$ ) suffices to detect and image the formation of xylem emboli in spruce and beech, and that 
these images can be used to quantify embolism formation by means of xylem vulnerability curves. While images with a matrix size of $32 \times 32$ (pixel sizes of 0.59 to $0.72 \mathrm{~mm}$ ) were not sufficient to spatially resolve the annual rings or finer details of the stem anatomy, they did permit an approximate localization of embolism formation (e.g. to differentiate older and younger xylem tissue on the basis of their position in the stem).

The ability to obtain meaningful data and vulnerability curves from quantitative but lowresolution images can be utilized in two ways. Firstly, by imaging at lower spatial resolution the acquisition time per image can be reduced dramatically. This enables continuous imaging at high time resolution, which may be beneficial to monitor rapid dynamics of embolism formation, for example during bench dehydration. Secondly, if the requirements for spatial resolution are relaxed, much simpler MRI devices can be used. Such imagers can be based on much weaker and therefore smaller and lighter permanent magnets, as well as on smaller and lighter gradient amplifiers. This has the potential to make non-invasive MR imaging of embolism formation much more affordable, and much more widely available to the plant hydraulics community.

\section{Ethics approval and consent to participate}

Not applicable.

\section{Consent for publication}

Not applicable.

\section{Availability of data and materials}

The datasets used and/or analysed during the current study are available from the corresponding author on reasonable request. 


\section{Competing interests}

371 The authors declare that they have no competing interests.

\section{Funding}

373 This work was supported by the German Research Foundation (DFG, grant number GRC81)

374 through the TUM International Graduate School of Science and Engineering (IGSSE) and the

375 IBG-2: Plant Sciences institute at the Forschungszentrum Jülich.

\section{Author Contributions}

$377 \mathrm{CW}$ and MM designed, built and programmed the magnetic resonance imager. MM performed 378 the experiment and analyzed the data. MM and CW wrote the manuscript with contributions 379 from PF.

\section{Acknowledgments}

381 We thank Johannes Kochs for his help in the design and construction of the magnet prototypes and the associated hardware, as well as Jonas Bühler for his help with the calculation of curve fitting errors. We thank Dr. Ute Sass-Klaassen for allowing access to the light microscopy facilities in her lab. Prof. Ulrich Schurr is gratefully acknowledged for making the presented research possible.

\section{References}

1. Nolf M, Lopez R, Peters JMR, Flavel RJ, Koloadin LS, Young IM, Choat B. Visualization of xylem embolism by X-ray microtomography: A direct test against hydraulic measurements. New Phytol. 2017;214:890-8. doi:10.1111/nph.14462. 
comparison of four techniques applied to a long-vesseled species. Plant Cell Environ. 2010;33:1502-12. doi:10.1111/j.1365-3040.2010.02160.x.

394

395

3. Knipfer T, Brodersen CR, Zedan A, Kluepfel DA, McElrone AJ. Patterns of droughtinduced embolism formation and spread in living walnut saplings visualized using X-ray microtomography. Tree Physiol. 2015;35:744-55. doi:10.1093/treephys/tpv040.

4. Choat B, Nolf M, Lopez R, Peters JMR, Carins-Murphy MR, Creek D, Brodribb TJ. Noninvasive imaging shows no evidence of embolism repair after drought in tree species of two genera. Tree Physiol 2018. doi:10.1093/treephys/tpy093.

5. Savi T, Miotto A, Petruzzellis F, Losso A, Pacilè S, Tromba G, et al. Drought-induced embolism in stems of sunflower: A comparison of in vivo micro-CT observations and destructive hydraulic measurements. Plant Physiol Biochem. 2017;120:24-9. doi:10.1016/j.plaphy.2017.09.017.

6. Petruzzellis F, Pagliarani C, Savi T, Losso A, Cavalletto S, Tromba G, et al. The pitfalls of in vivo imaging techniques: Evidence for cellular damage caused by synchrotron X-ray computed micro-tomography. New Phytol. 2018;220:104-10. doi:10.1111/nph.15368.

7. Dhondt S, Vanhaeren H, van Loo D, Cnudde V, Inzé D. Plant structure visualization by high-resolution X-ray computed tomography. Trends Plant Sci. 2010;15:419-22. doi:10.1016/j.tplants.2010.05.002.

8. Nagata A, Kose K, Terada Y. Development of an outdoor MRI system for measuring flow in a living tree. J Magn Reson. 2016;265:129-38. doi:10.1016/j.jmr.2016.02.004.

9. Windt CW, Gerkema E, van As H. Most water in the tomato truss is imported through the xylem, not the phloem: A nuclear magnetic resonance flow imaging study. Plant Physiol. 2009;151:830-42. doi:10.1104/pp.109.141044.

10. van As H, Scheenen T, Vergeldt FJ. MRI of intact plants. Photosyn Res. 2009;102:21322. doi:10.1007/s11120-009-9486-3. 
11. Meixner M, Tomasella M, Foerst P, Windt CW. A small-scale MRI scanner and complementary imaging method to visualize and quantify xylem embolism formation. New Phytol 2020. doi:10.1111/nph.16442.

12. Clearwater MJ, Clark CJ. In vivo magnetic resonance imaging of xylem vessel contents in woody lianas. Plant Cell Environ. 2003;26:1205-14. doi:10.1046/j.13653040.2003.01042.x.

13. Holbrook NM, Ahrens E. T., Burns M. J., Zwieniecki MA. In Vivo Observation of Cavitation and Embolism Repair Using Magnetic Resonance Imaging. Plant Physiol. 2001;126:27-31. doi:10.1104/pp.126.1.27.

14. Hochberg U, Albuquerque C, Rachmilevitch S, Cochard H, David-Schwartz R, Brodersen $\mathrm{CR}$, et al. Grapevine petioles are more sensitive to drought induced embolism than stems: Evidence from in vivo MRI and microcomputed tomography observations of hydraulic vulnerability segmentation. Plant Cell Environ. 2016;39:1886-94. doi:10.1111/pce.12688.

15. Bouda M, Windt CW, McElrone AJ, Brodersen CR. In vivo pressure gradient heterogeneity increases flow contribution of small diameter vessels in grapevine. Nature communications. 2019;10:1-10. doi:10.17605/OSF.IO/EY2TJ.

16. Scheenen T, Anneriet Heemskerk, Andrie de Jager, Frank Vergeldt, Henk Van As. Functional Imaging of Plants: A Nuclear Magnetic Resonance Study of a Cucumber Plant 2002. doi:10.1016/S0006-3495(02)75413-1.

17. Ilja Kaufmann, Thomas Schulze-Till, Heike U. Schneider, Ulrich Zimmermann, Peter Jakob. Functional repair of embolized vessels in maize roots after temporal drought stress, as demonstrated by magnetic resonance imaging. New Phytol. 2009;184:245-56. doi:10.1111/j.1469-8137.2009.02919.x. 
18. Zwieniecki MA, Melcher PJ, Ahrens ET. Analysis of spatial and temporal dynamics of xylem refilling in Acer rubrum L. using magnetic resonance imaging. Front Plant Sci. 2013;4:265. doi:10.3389/fpls.2013.00265.

19. Fukuda K, Kawaguchi D, Aihara T, Ogasa MY, Miki NH, Haishi T, Umebayashi T. Vulnerability to cavitation differs between current-year and older xylem: Non-destructive observation with a compact magnetic resonance imaging system of two deciduous diffuse-porous species. Plant Cell Environ. 2015;38:2508-18. doi:10.1111/pce.12510.

20. Umebayashi T, Morita T, Utsumi Y, Kusumoto D, Yasuda Y, Haishi T, Fukuda K. Spatial distribution of xylem embolisms in the stems of Pinus thunbergii at the threshold of fatal drought stress. Tree Physiol. 2016;36:1210-8. doi:10.1093/treephys/tpw050.

21. Meixner M, Kochs J, Foerst P, Windt CW. An integrated magnetic resonance plant imager. 2020. In prep.

22. Pammenter NW, van der Willigen C. A mathematical and statistical analysis of the curves illustrating vulnerability of xylem to cavitation. Tree Physiol. 1998;18:589-93. doi:10.1093/treephys/18.8-9.589.

23. Brown RW, Cheng Y-CN, Haacke EM, Thompson MR, Venkatesan R. Magnetic resonance imaging: Physical principles and sequence design: John Wiley \& Sons; 2014.

24. Windt CW, Soltner H, van Dusschoten D, Blümler P. A portable Halbach magnet that can be opened and closed without force: The NMR-CUFF. J Magn Reson. 2011;208:27-33. doi:10.1016/j.jmr.2010.09.020.

25. Ma J, Slavens Z, Sun W, Bayram E, Estowski L, Hwang K-P, et al. Linear phase-error correction for improved water and fat separation in dual-echo dixon techniques. Magn Reson Med. 2008;60:1250-5. doi:10.1002/mrm.21747.

26. Edzes HT, van Dusschoten D, van As H. Quantitative T2 imaging of plant tissues by means of multi-echo MRI microscopy. Magnetic Resonance Imaging. 1998;16:185-96. doi:10.1016/S0730-725X(97)00274-9. 
27. Press WH. Numerical recipes in C++: The art of scientific computing. 2nd ed.

$$
\text { Cambridge, UK: Cambridge University Press; } 2002 .
$$

28. Gärtner H, Schweingruber FH. Microscopic preparation techniques for plant stem analysis: Verlag Dr. Kessel; 2013.

29. Umebayashi T, Fukuda K, Haishi T, Sotooka R, Zuhair S, Otsuki K. The developmental process of xylem embolisms in pine wilt disease monitored by multipoint imaging using compact magnetic resonance imaging. Plant Physiol. 2011;156:943-51. doi:10.1104/pp.110.170282.

30. Merela M, Sepe A, Oven P, Sersa I. Three-dimensional in vivo magnetic resonance microscopy of beech (Fagus sylvatica L.) wood. MAGMA. 2005;18:171-4.

$$
\text { doi:10.1007/s10334-005-0109-5. }
$$

31. Gebauer R, Plichta R, Urban J, Volařík D, Hájíčková M. The resistance and resilience of European beech seedlings to drought stress during the period of leaf development. Tree Physiol 2020. doi:10.1093/treephys/tpaa066.

32. Pallardy SG. Physiology of woody plants: Academic Press; 2010.

33. Caquet B, Barigah TS, Cochard H, Montpied P, Collet C, Dreyer E, Epron D. Hydraulic properties of naturally regenerated beech saplings respond to canopy opening. Tree Physiol. 2009;29:1395-405. doi:10.1093/treephys/tpp067.

34. Aranda I, Cano FJ, Gascó A, Cochard H, Nardini A, Mancha JA, et al. Variation in photosynthetic performance and hydraulic architecture across European beech (Fagus sylvatica L.) populations supports the case for local adaptation to water stress. Tree Physiol. 2015;35:34-46. doi:10.1093/treephys/tpu101.

35. Lemoine D, Cochard H, Granier A. Within crown variation in hydraulic architecture in beech (Fagus sylvatica L): Evidence for a stomatal control of xylem embolism. Ann. For. Sci. 2002;59:19-27. doi:10.1051/forest:2001002. 
36. Herbette S, Wortemann R, Awad H, Huc R, Cochard H, Barigah TS. Insights into xylem vulnerability to cavitation in Fagus sylvatica L.: Phenotypic and environmental sources of variability. Tree Physiol. 2010;30:1448-55. doi:10.1093/treephys/tpq079.

37. Schuldt B, Knutzen F, Delzon S, Jansen S, Müller-Haubold H, Burlett R, et al. How adaptable is the hydraulic system of European beech in the face of climate change-related precipitation reduction? New Phytol. 2016;210:443-58. doi:10.1111/nph.13798.

38. Stojnic S, Suchocka M, Benito-Garzón M, Torres-Ruiz JM, Cochard H, Bolte A, et al. Variation in xylem vulnerability to embolism in European beech from geographically marginal populations. Tree Physiol. 2018;38:173-85. doi:10.1093/treephys/tpx128.

39. Tomasella M, Beikircher B, Häberle K-H, Hesse B, Kallenbach C, Matyssek R, Mayr S. Acclimation of branch and leaf hydraulics in adult Fagus sylvatica and Picea abies in a forest through-fall exclusion experiment. Tree Physiol. 2018;38:198-211. doi:10.1093/treephys/tpx140.

40. Mayr S, Rothart B, Dämon B. Hydraulic efficiency and safety of leader shoots and twigs in Norway spruce growing at the alpine timberline. J Exp Bot. 2003;54:2563-8. doi:10.1093/treephys/11.1.73.

41. Chmura DJ, Guzicka M, McCulloh KA, Żytkowiak R. Limited variation found among Norway spruce half-sib families in physiological response to drought and resistance to embolism. Tree Physiol. 2016;36:252-66. doi:10.1093/treephys/tpv141.

42. Cochard H. Vulnerability of several conifers to air embolism. Tree Physiol. 1992;11:7383. doi:10.1093/treephys/11.1.73.

\section{Figures}

Figure 1. Sequence of continuous imaging at three spatial resolutions. Blocks of three matrix sizes were repeated, resulting in three time series of images, each 25.3 minutes apart. 
Figure 2. Water content $(A)$, relaxation time $\left(T_{2}\right)$ and $A * T_{2}$ product images of a well-watered spruce (a) and beech (b) sapling at matrix sizes $32 \times 32,64 \times 64$, and $128 \times 128$. At the different matrix sizes 8, 16 and 32 acquisitions were averaged for spruce and 4, 8 and 16 for beech, respectively. Total acquisition times were 7.0, 28.8, and 116.8 minutes for spruce and 3.5, 14.4 and 58.4 minutes, respectively.

Figure 3. $\mathrm{A} * \mathrm{~T}_{2}$ product maps of xylem embolism formation in spruce (a) and beech (b). Images were acquired with matrix sizes of $32 \times 32,64 \times 64$ and $128 \times 128$ pixels, shown in dependence of time and water potential (WP). The concomitant water content and $\mathrm{T}_{2}$ images are provided in Additional file 2. Imaging continuously allowed for resolving finer spatio-temporal details of embolism formation. Shown are the first emboli forming in spruce (c), and the completion of xylem dry-down in beech (d). In all panels (a-d), the difference in image intensity between the first and the last image is shown in the rightmost position $\left(\Delta_{-}\right)$.

Figure 4. Light microscopy of spruce (Picea abies, a) and beech (Fagus sylvatica, b). The thin sections here shown were obtained from the same position where MR imaging was done. Insets illustrate the difference in pixel sizes between the three spatial resolutions (matrix sizes) used in the MR imaging experiments.

Figure 5. Vulnerability curves (VCs) obtained on the basis of binarization of $\mathrm{A}^{*} \mathrm{~T}_{2}$ product maps. The matrix size of the images were varied $(32 \times 32,64 \times 64$, and $128 \times 128)$, while the field of view was held constant. A subset of the binarization masks that the VC's are based on is shown, together with the corresponding water potentials (WP), in panel (a) for spruce and panel (b) beech. The resulting VCs are shown in panels (c), (e) and (g) for spruce; and in panels (d), (f) and (h) for beech.

\section{Additional files}


539 Additional file 1. First echo images of well-watered spruce (a) and beech (b), acquired with an

540 MSE imaging sequence, illustrating the dependency of image quality and signal-to-noise ratio

541 on matrix size and the number of acquisitions averaged (numAcq). The respective SNR is

542 printed under each image.

543 Additional file 2: Water content (A) and $\mathrm{T}_{2}$ maps of progressive xylem embolism formation in 544 spruce $(a, b)$ and beech $(c, d)$, acquired with matrix sizes of 32x32, 64x64 and 128x128 pixels, 545 and shown in dependence of time and water potential (WP). In all panels (a-d), the difference 546 in image intensity between the first and the last image is shown in the rightmost position $\left(\Delta_{-}\right)$.

547 Additional files 3. Animated illustration of the xylem dry-down of spruce (a) and beech (b).

548 Water content $(\mathrm{A}), \mathrm{T}_{2}$ and $\mathrm{A} * \mathrm{~T}_{2}$ maps are displayed at different imaging matrices $(32 \times 32$, $54964 \times 64$ and $128 \times 128$ ), all with the same field of view. In the upper right corner the time and 550 water potential values corresponding with each image are shown. 
Figures

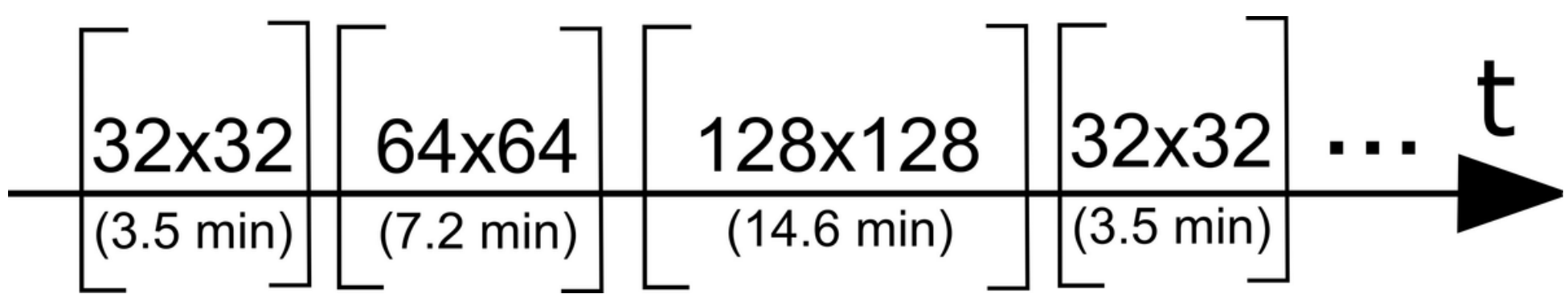

Figure 1

Sequence of continuous imaging at three spatial resolutions. Blocks of three matrix sizes were repeated, resulting in three time series of images, each 25.3 minutes apart. 


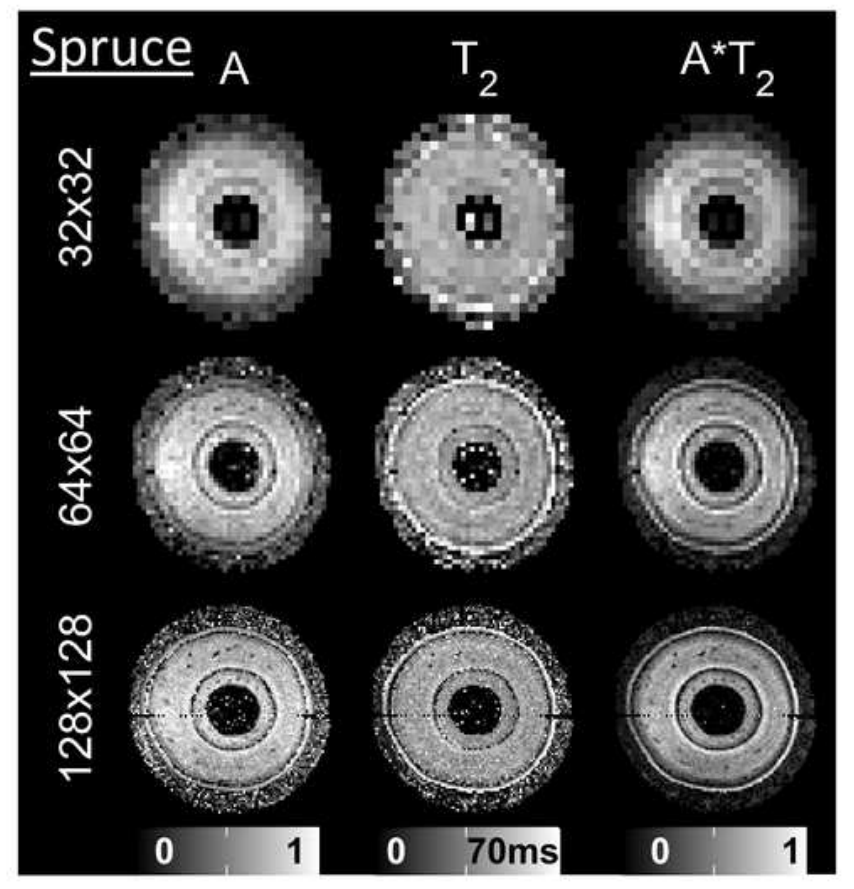

(a)

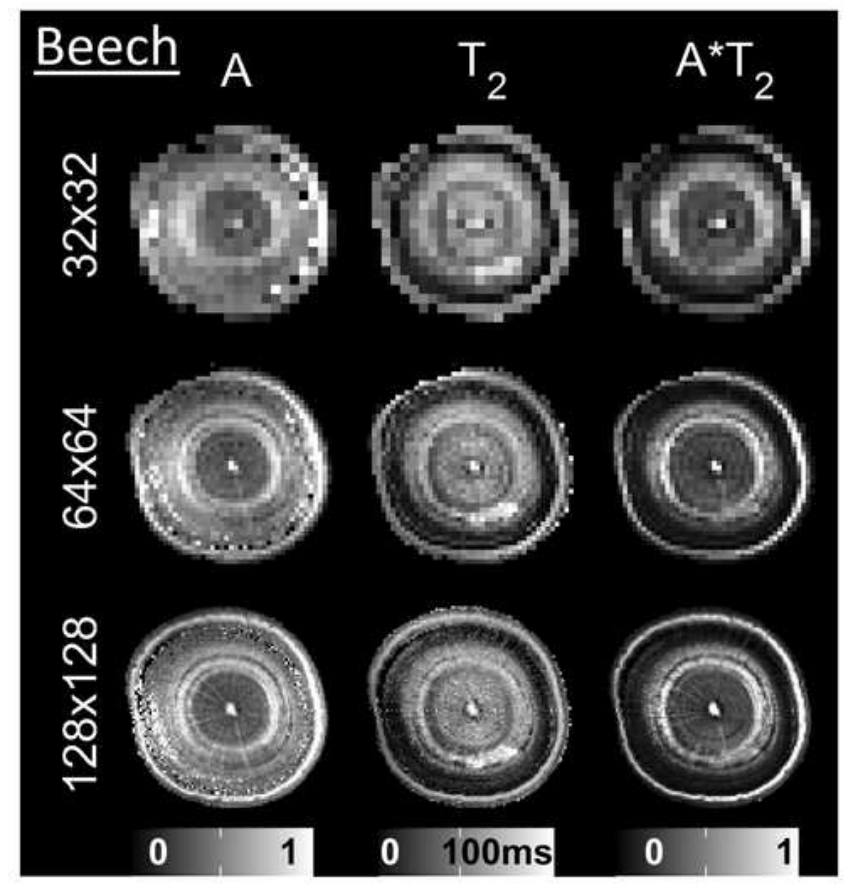

(b)

Figure 2

Water content (A), relaxation time (T2) and $A^{\star} T 2$ product images of a well-watered spruce (a) and beech (b) sapling at matrix sizes $32 \times 32,64 \times 64$, and $128 \times 128$. At the different matrix sizes 8,16 and 32 acquisitions were averaged for spruce and 4,8 and 16 for beech, respectively. Total acquisition times were $7.0,28.8$, and 116.8 minutes for spruce and $3.5,14.4$ and 58.4 minutes, respectively. 


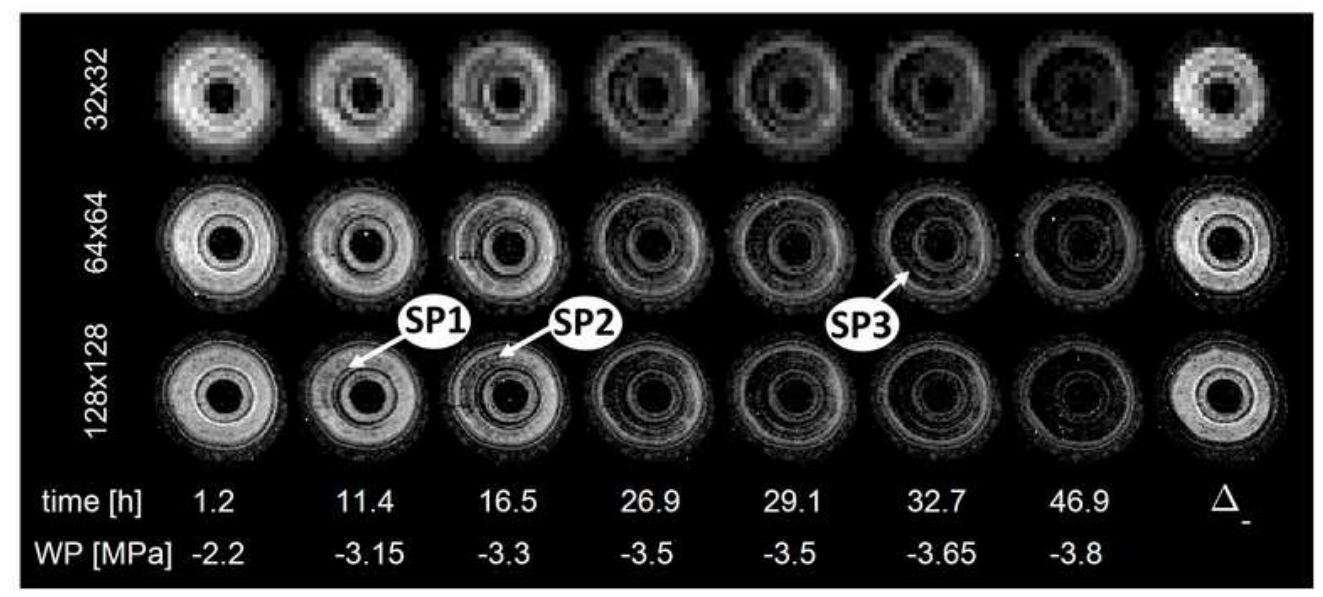

(a)

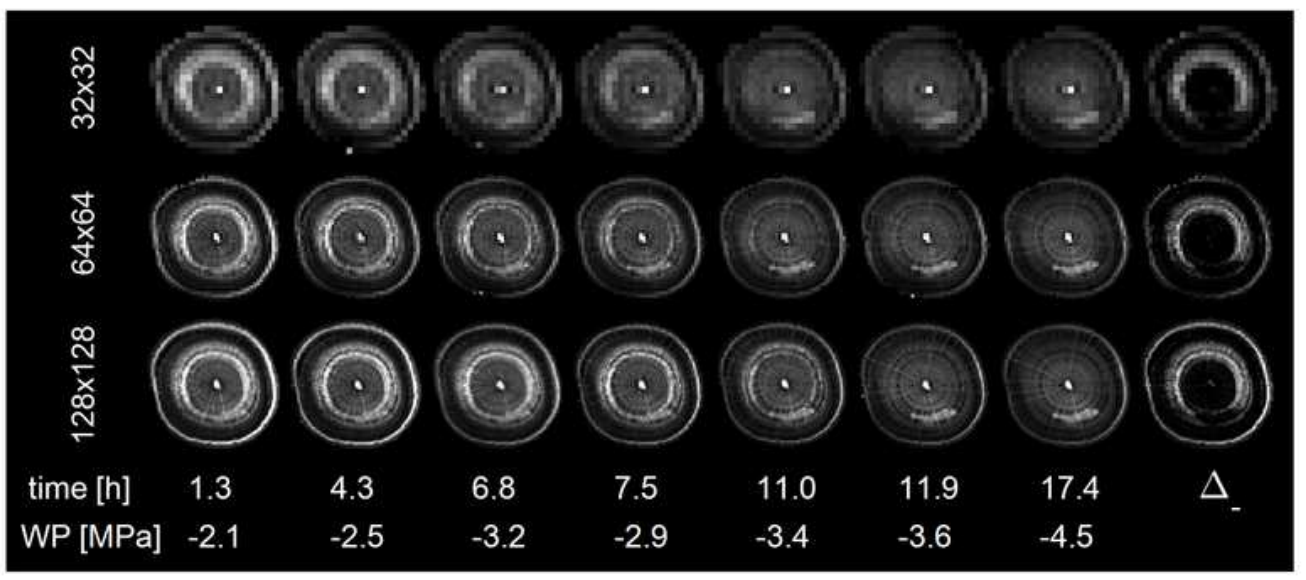

(b)

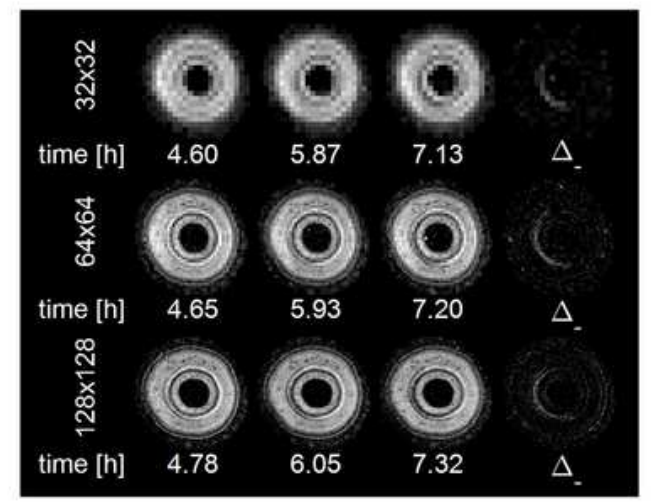

(c)

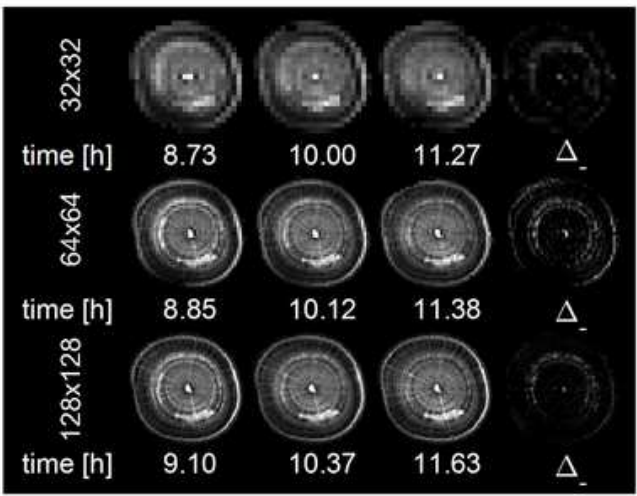

(d)

\section{Figure 3}

$A^{*} T 2$ product maps of xylem embolism formation in spruce (a) and beech (b). Images were acquired with matrix sizes of $32 \times 32,64 \times 64$ and $128 \times 128$ pixels, shown in dependence of time and water potential (WP). The concomitant water content and T2 images are provided in Additional file 2. Imaging continuously allowed for resolving finer spatio-temporal details of embolism formation. Shown are the first emboli forming in spruce (c), and the completion of xylem dry-down in beech (d). In all panels (a-d), 
the difference in image intensity between the first and the last image is shown in the rightmost position $\left(\Delta_{-}-\right)$.
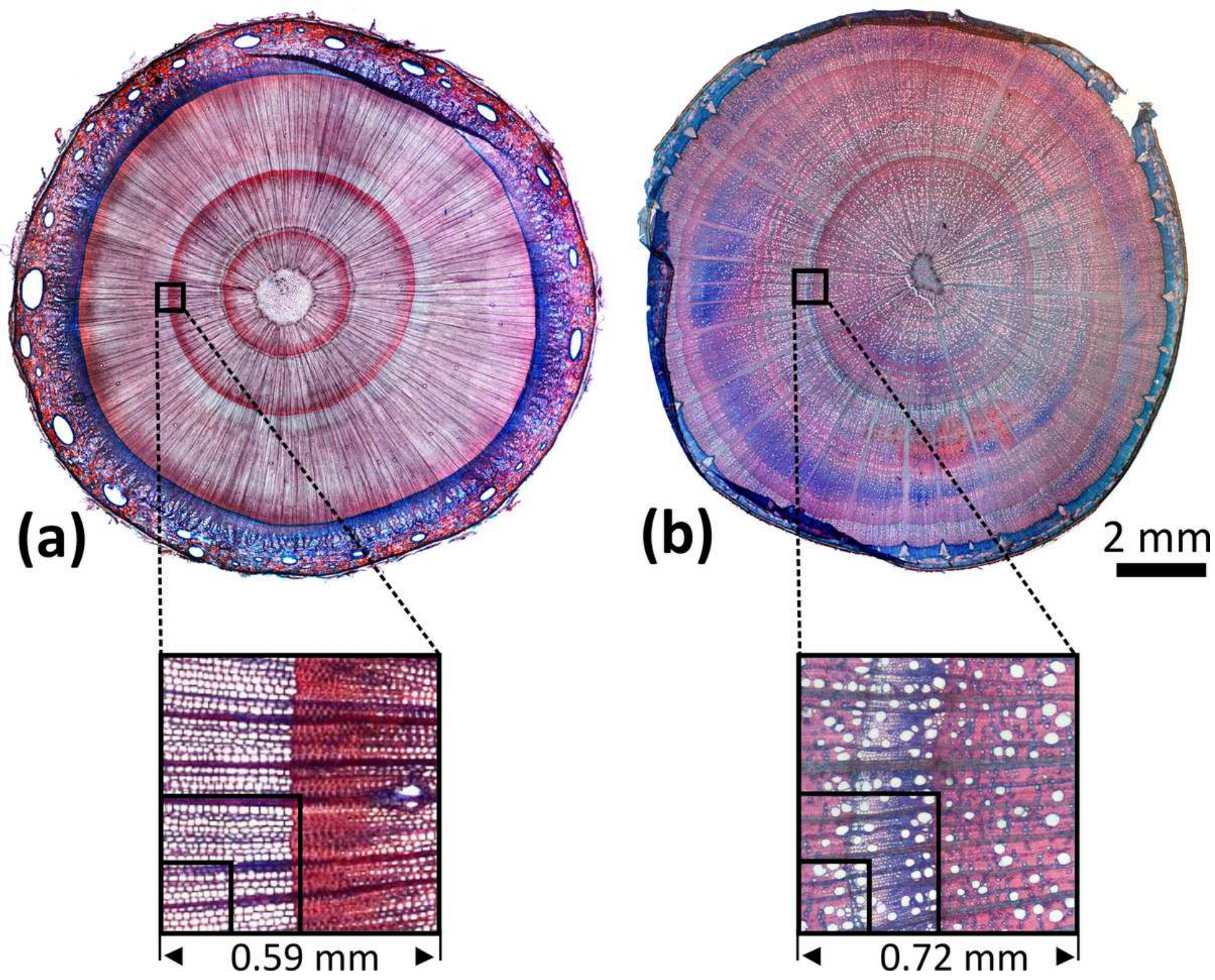

Figure 4

Light microscopy of spruce (Picea abies, a) and beech (Fagus sylvatica, b). The thin sections here shown were obtained from the same position where MR imaging was done. Insets illustrate the difference in pixel sizes between the three spatial resolutions (matrix sizes) used in the MR imaging experiments. 


\section{Spruce}

\section{Beech}

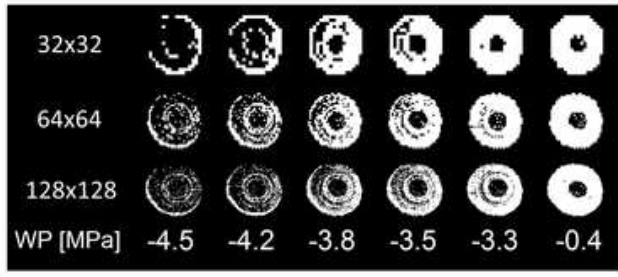

(a)

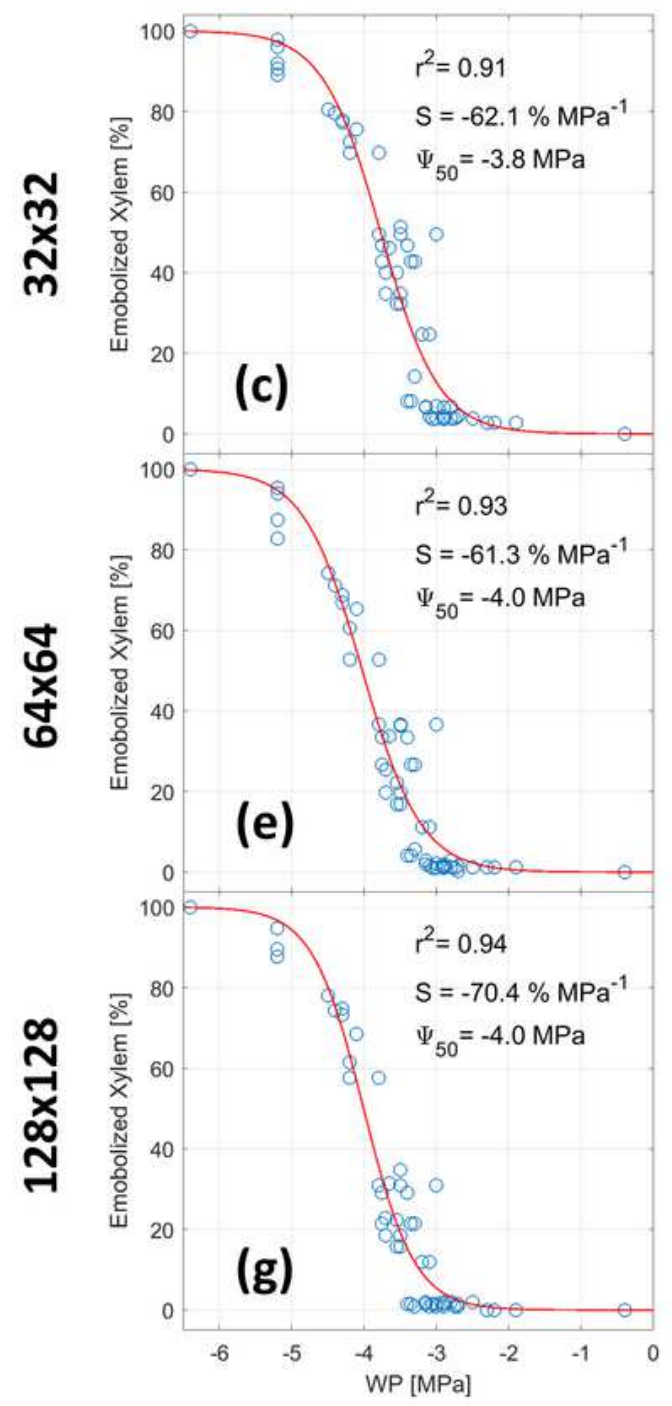

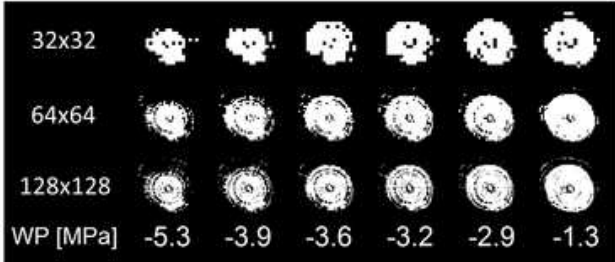

(b)

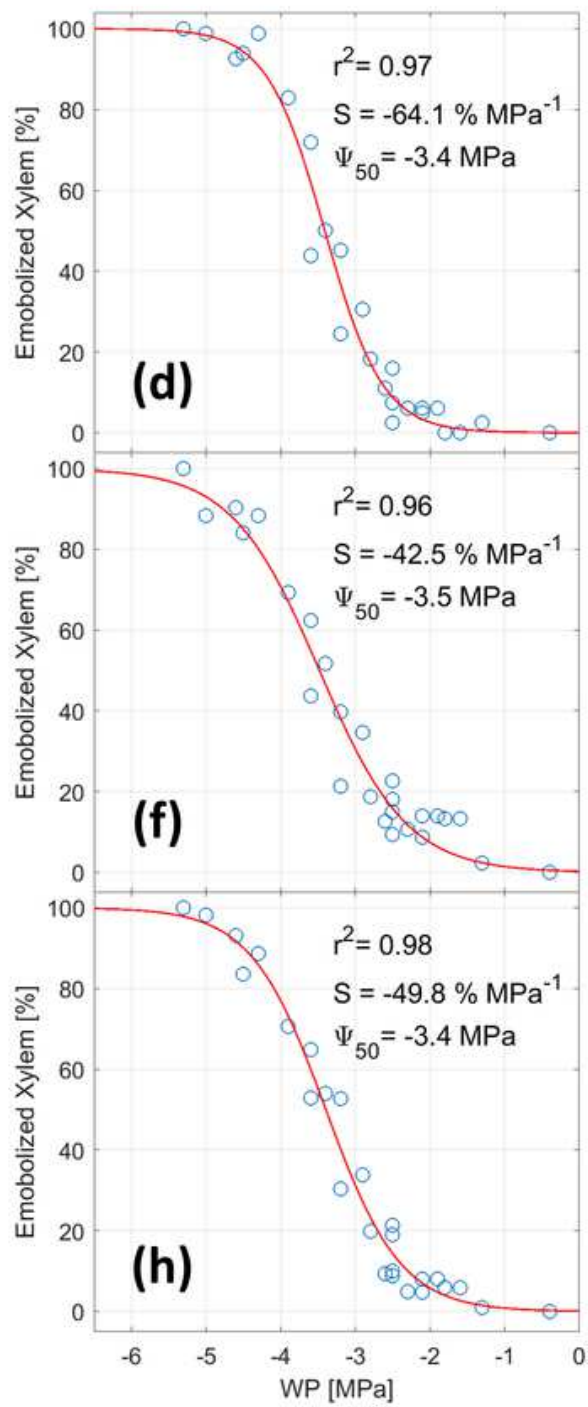

\section{Figure 5}

Vulnerability curves (VCs) obtained on the basis of binarization of $A^{\star} T 2$ product maps. The matrix size of the images were varied $(32 \times 32,64 \times 64$, and $128 \times 128)$, while the field of view was held constant. A subset of the binarization masks that the VC's are based on is shown, together with the corresponding water potentials (WP), in panel (a) for spruce and panel (b) beech. The resulting VCs are shown in panels (c), (e) and $(\mathrm{g})$ for spruce; and in panels $(\mathrm{d}),(\mathrm{f})$ and $(\mathrm{h})$ for beech. 


\section{Supplementary Files}

This is a list of supplementary files associated with this preprint. Click to download.

- Additionalfile1.png

- Additionalfile2.png

- Additionalfile3aspruce.avi

- Additionalfile3bbeech.avi 